$\S=-1$

\title{
Brinkman Flow Generated by the Rectilinear Oscillations of an Oblate Spheroid Along its Axis of Symmetry
}

\author{
Satish Kumar. ${ }^{1 *}$, S. K. Vali ${ }^{2}$ \\ ${ }^{I}$ Dept.of Mathematics, Koneru Lakshmaiah Education Foundation, Vaddeswaram- 522502, India \\ ${ }^{2}$ Depart of Mathematics, Jawaharlal Nehru Technological University College of Engineerig,Vizianagaram- 535 002, India \\ *Corresponding author E-mail: satish9441321888@gmail.com
}

\begin{abstract}
In this paper, we consider an impervious Oblate spheroid placed in a fully saturated porous medium, where in the flow is governed by Brinkmann flow equation. We assume that the spheroid is performing rectilinear harmonic oscillations along the axis of symmetry with a speed $u$. The flow is studied under the Stokesian approximation. The expressions for the velocity and pressure fields are obtained in terms of Legendre functions, associated Legendre functions and Radial and Angular spheroidal wave functions. We obtain an expression for the drag experienced by the spheroid, and numerically study its variation with respect to the flow parameters and display its variation through graphs.
\end{abstract}

Keywords: Rectilinear oscillations Oblate spheroid, porous medium, Stokesian assumption, Brinkman model, velocity, pressure, drag

\section{Introduction}

A class of problems of interest for researches in fluid mechanics over years is constituted by the oscillating flows generated by the rectilinear or rotary oscillations of axially symmetric bodies in an infinite expanse of viscous liquid which is otherwise at rest. When we review the equations describing such flows, we recognize that the equations are not solvable unless some simplifying assumptions are made. One such simplifying assumption is that nearer to the oscillating body the viscous terms in the governing Navier-Stokes equation predominate over the acceleration terms. Neglecting the acceleration terms ( which are also called inertial terms) we get a linearized version of the equations for the determination of the flow field. The assumption is referred to as Stokesian assumption and this is allowable when the maximum distance travelled by a fluid particle nearer to the oscillating body over one period of motion is smaller than the characteristic length corresponding to the body as observed by Pozrikidis [1]. Sir G.J.Stokes about one and half centuries back studied the flow generated by the oscillations of a pendulum under this assumption [2].

In 1950's Kanwal studied the oscillatory flows generated by the rotary and longitudinal oscillations of axisymmetric bodies in a viscous liquid which is otherwise at rest. The bodies considered there in are sphere, spheroid ( both Prolate and Oblate), elliptic cylinder and a flat plate [3]. In some incompressible fluids of different kinds are also studied in $[4,5,6]$. The authors have earlier studied the Stokes flow past a Prolate spheroid and Oblate spheroid in Brinkmann medium, Rectilinear oscillations of a prolate spheroid in a saturated porous medium [7,8].In this paper we propose to study the rectilinear oscillations of an oblate spheroid placed in a porous medium where in the flow field is governed by Brinkmann equations. We obtain the components of velocity and the drag experienced by the spheroid and study the variation of the drag numerically with respect to the porosity parameter and size of the spheroid and frequency parameter.

\section{Mathematical Formulation}

Consider the flow of an incompressible viscous fluid in a (saturated) porous medium due to the rectilinear oscillations of an Oblate spheroid along its axis of symmetry. Let the spheroid oscillate with speed of oscillation $\mathrm{Ue}^{\mathrm{i} \omega \mathrm{t}}$. The schematic diagram of the flow as shown in Figure.(1)

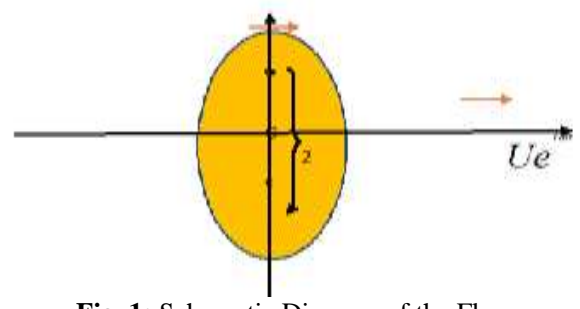

Fig. 1: Schematic Diagram of the Flow

Let $\mathrm{c}$ be the semi focal distance of the ellipse generating the oscillating oblate spheroid. Equations describing the viscous fluid flow in the porous region are $\nabla \cdot \bar{q}=0$

$\rho \frac{d \bar{q}}{d t}=-\nabla p-\mu \nabla \times(\nabla \times \bar{q})-\frac{\mu}{k^{*}} \bar{q}$

where $\mathrm{k}^{*}$ is the permeability of the porous medium. Let $(\xi, \eta, \phi)$ denote a spheroidal system of coordinates with $\left(\overline{e_{\xi}}, \overline{\mathrm{e}_{\eta}}, \overline{\mathrm{e}_{\phi}}\right)$ as unit base vectors and $\left(\mathrm{h}_{1}, \mathrm{~h}_{2}, \mathrm{~h}_{3}\right)$ as a scale factors. The flow generat- 
ed by the oscillations of the spheroid is assumed to be axially symmetric and in view of this, all the flow field variables are independent of the angular coordinate variable $\phi$. we assume that the velocity

$$
\bar{q}=\bar{q}(\xi, \eta) e^{i \omega t}=\left[\mathrm{u}(\xi, \eta) \overline{e_{\xi}}+v(\xi, \eta) \bar{e}_{\eta}\right] e^{i \omega t}
$$

In view of the continuity equation (5. 1), we introduce stream function $\psi(\xi, \eta) e^{i \omega t}$ through

$$
h_{2} h_{3} u=-\frac{\partial \psi}{\partial \eta} ; h_{1} h_{3} v=\frac{\partial \psi}{\partial \xi}
$$

Let the pressure $\mathrm{p}$ be written as $p(\xi, \eta, t)=P(\xi, \eta) \mathrm{e}^{i \omega t}$

Using (3), (4) and (5) in equation (2) after cancellation of $e^{i \omega t}$ and that equation by eliminating pressure we get

$$
E^{4} \psi-\left(\frac{1}{k^{*}}+\frac{i \rho \omega}{\mu}\right) E^{2} \psi=0
$$

Here $E^{2}$ is the Stokes steam function operator given by

$E^{2}=\frac{h_{3}}{h_{1} h_{2}}\left(\frac{\partial}{\partial \xi}\left(\frac{h_{2}}{h_{1} h_{3}} \frac{\partial}{\partial \xi}\right)-\frac{\partial}{\partial \eta}\left(\frac{h_{1}}{h_{2} h_{3}} \frac{\partial}{\partial \eta}\right)\right)$

Introducing

$$
\frac{\alpha^{2}}{c^{2}}=\frac{i \rho \omega}{\mu}+\frac{1}{k^{*}}
$$

Here we note that $\alpha$ is a non-dimensional parameter depending on the oscillation parameter PT and porosity parameter Pe defined by $P T=\frac{\rho \omega c^{2}}{\mu} ; \mathrm{Pe}=\frac{k^{*}}{c^{2}}$

Here, $\alpha$ is genuinely complex . $\alpha$ is real if $\boldsymbol{P T}=\mathbf{O}$ ie., if there is no oscillation. $\alpha^{2}$ is purely imaginary if we have nonporous medium. For numerical work we take $\alpha$ as the square root of $i P T+\frac{1}{P e}$ whose real part is +ve.

(6) can be written as

$$
E^{2}\left(E^{2}-\frac{\alpha^{2}}{c^{2}}\right) \psi=0
$$

\section{Solution of $\psi$ :}

Let $\psi=\psi_{1}+\psi_{2}$ be the solution of (10) is obtained by super imposing the solution of $E^{2} \psi=0$ and $\left(E^{2}-\frac{\alpha^{2}}{c^{2}}\right) \psi=0$

We note that $\psi$ has satisfy the following conditions

1.Far away from the oscillating body there is practically no flow and the function $\psi \rightarrow 0$

2.At the boundary of oscillating body we adopt the no slip condition: the velocity of a fluid element on the body equals that of the oscillating body

Let us introduce $\tau=\sinh \xi ; t=\cos \eta$

With this, the scale factors of the coordinate system turn out to be $h_{1}=h_{2}=c \sqrt{\tau^{2}+t^{2}} \quad h_{3}=c \sqrt{\left(\tau^{2}+1\right)\left(1-t^{2}\right)}$

Hence (7) Becomes

$$
E^{2}=\frac{1}{\mathrm{c}^{2}\left(\tau^{2}+\mathrm{t}^{2}\right)}\left[\left(\tau^{2}+1\right) \frac{\partial^{2}}{\partial \tau^{2}}+\left(1-\mathrm{t}^{2}\right) \frac{\partial^{2}}{\partial t^{2}}\right]
$$

3.1 Solution of $E^{2} \psi_{1}=0$ which satisfies the regularity condition in the flow field and vanishing of velocity far away from the body is seen to be

$$
\psi_{1}=U c^{2} \sqrt{\left(\tau^{2}+1\right)\left(1-t^{2}\right)} \sum_{n=1}^{\infty} A_{n} Q_{n}^{(1)}(\mathrm{i} \tau) p_{n}^{(1)}(t)
$$

Here $Q_{n}^{(1)}(\mathrm{s})$ and $P_{n}^{(1)}(t)$ are associated Legendre functions of the second and first kind respectively, and is given by

$$
Q^{(1)}{ }_{n}(i \tau)=\sqrt{\left(\tau^{2}+1\right)} \frac{d}{d \tau} Q_{n}(i \tau), P_{n}^{(1)}(\mathrm{t})=\sqrt{\left(1-\mathrm{t}^{2}\right.} \frac{d}{d t} P_{n}(\mathrm{t})
$$

3.2 Solution of $\left(E^{2}-\frac{\alpha^{2}}{c^{2}}\right) \psi_{2}=0$ which vanishes far away from the body and is regular on the axis of symmetry is given by

$$
\psi_{2}(\tau, t)=U c^{2} \sqrt{\left(\tau^{2}+1\right)\left(1-t^{2}\right)} \sum_{n=1}^{\infty} B_{m} R_{1 m}^{(3)}(\mathrm{i} \alpha, \tau) S_{1 m}^{(1)}(\mathrm{i} \alpha, t)
$$

where $R_{1 m}^{(3)}(\mathrm{i} \alpha, \tau) ; S_{1 m}^{(1)}(\mathrm{i} \alpha, t)$ are oblate radial and angular wave functions respectively [7].

These are respectively same as the prolate radial and prolate angular functions $R_{1 n}^{(3)}(\alpha, i \tau)$, and $S_{1 n}^{(1)}(\alpha, t)$ which are given by

$$
\begin{aligned}
R_{1 n}^{(3)}(\alpha, \mathrm{i} \tau) & =\left[i^{n+2} \sum_{r=0,1}^{\infty^{\prime}}(r+1)(r+2) d_{r}^{1 m}(\alpha)\right]^{-1} \\
& \times \sqrt{\frac{2}{\pi \alpha}}\left(\frac{\tau^{2}+1}{\tau^{3}}\right)^{\frac{1}{2}} \sum_{r=0,1}^{\infty}(r+1)(r+2) d_{r}^{1 m}(\alpha) \mathrm{K}_{r+\frac{3}{2}}(\alpha \tau)
\end{aligned}
$$

$S_{1 n}^{1}(\alpha, t)=\sum_{r=0,1}^{\infty} d_{r}^{1 m}(\alpha) \mathrm{P}_{r+1}^{(1)}(\mathrm{t})$

Here $K_{r+\frac{3}{2}}(\alpha \tau)$ is modified Bessel function of second kind, and $\mathrm{P}_{r+1}^{(1)}(\mathrm{t})$ is the associated Legendre function of the first kind. The coefficients $\mathrm{d}_{r}^{1 n}(i \alpha)$ in the above expansions are constants depending on the parameter $i \alpha$ and the suffix r has the value $1,3,5,--$ --- or $0,2,4,----d e p e n d i n g$ upon the odd or even nature of $(n+1)$ as in [7].

The solution of (10) which vanishes far away from the oscillating body and is regular in the flow region is given by

$$
\begin{gathered}
\psi(\tau, t)=U c^{2} \sqrt{\left(\tau^{2}+1\right)\left(1-t^{2}\right)} \sum_{n=1}^{\infty} A_{n} Q_{n}^{(1)}(\mathrm{i} \tau) p_{n}^{(1)}(t) \\
+U c^{2} \sqrt{\left(\tau^{2}+1\right)\left(1-t^{2}\right)} \sum_{m=1}^{\infty} B_{m} R_{1 m}^{(3)}(\alpha, \mathrm{i} \tau) S_{1 m}^{(1)}(\alpha, t)
\end{gathered}
$$

Here $\left\{A_{n}\right\}$ and $\left\{B_{n}\right\}$ are all determined subject to the boundary conditions. 


\section{Boundary Conditions}

In view of the no slip conditions on the boundary, we require that

$$
\begin{aligned}
& \mathrm{u}\left(\tau_{0}, \mathrm{t}\right)=\frac{U \sqrt{\left(\tau_{0}^{2}+1\right)} t}{\sqrt{\left(\tau_{0}^{2}+\mathrm{t}^{2}\right)}} \\
& \mathrm{v}\left(\tau_{0}, \mathrm{t}\right)=-\frac{U \sqrt{\left(1-t^{2}\right)} \tau_{0}}{\sqrt{\left(\tau_{0}^{2}+\mathrm{t}^{2}\right)}}
\end{aligned}
$$

Rewriting these in terms of $\psi$, we need

$$
\begin{aligned}
& \frac{\partial \psi}{\partial t}_{\tau=\tau_{0}}=U c^{2}\left(\tau_{0}^{2}+1\right) \mathrm{t} \\
& \frac{\partial \psi}{\partial \tau}_{\tau=\tau_{0}}=-U c^{2}\left(1-\mathrm{t}^{2}\right) \tau_{0}
\end{aligned}
$$

Using (19) and (22), and later multiply with $p_{n}(t)$,integrate from -1 to 1 w.r.t. t. On simplification we get

$$
A_{n} Q_{n}^{(1)}(\mathrm{i} \tau)+\sum_{m=1}^{\infty} B_{m} R_{1 m}^{(3)}(i \alpha, \tau) d_{n-1}^{1 m}(i \alpha)=-\frac{1}{2} \sqrt{\left(\tau_{0}^{2}+1\right)} \delta_{n 1}
$$

Using (19) and (23), and later multiply with $p_{n}(t)$,integrate from -1 to 1 w.r.t. t. On simplification we get

$$
\begin{gathered}
A_{n} n(n+1) Q_{n}(\mathrm{i} \tau)+\sum_{m=1}^{\infty} B_{m} \frac{d}{d \tau}\left(\sqrt{\left(\tau^{2}+1\right)} R_{1 m}^{(3)}(i \alpha, \tau)\right) d_{n-1}^{1 m}(i \alpha) \\
=-\tau_{0} \delta_{n 1}
\end{gathered}
$$

Eliminating An from (24) and (25) We get

$$
\begin{aligned}
\sum_{m=1}^{\infty} B_{m} d_{n-1}^{1 m}(i \alpha)\left(Q_{n}^{(1)}(\mathrm{i} \tau)\right. & \left.\frac{d}{d \tau}\left(\sqrt{\left(\tau^{2}+1\right)} R_{1 m}^{(3)}(i \alpha, \tau)\right)-n(n+1) Q_{n}(\mathrm{i} \tau) R_{1 m}^{(3)}(i \alpha, \tau)\right) \\
& =\left(Q_{1}(\mathrm{i} \tau) \sqrt{\left(\tau_{0}^{2}+1\right)}-\tau_{0} \sqrt{\left(\tau_{0}^{2}+1\right)} \frac{d}{d \tau} Q_{1}(\mathrm{i} \tau)\right) \delta_{n 1} \\
& =\sqrt{\left(\tau_{0}^{2}+1\right)}\left(Q_{1}(\mathrm{i} \tau)-\tau_{0} \frac{d}{d \tau} Q_{1}(\mathrm{i} \tau)\right) \delta_{n 1} \\
& =\frac{1}{\sqrt{\left(\tau_{0}^{2}+1\right)}} \delta_{n 1}
\end{aligned}
$$

Hence $B_{m}$ 's can be determined by using the infinite system of non homogeneous linear equations in $B_{m}$ given by

$$
\sum_{m=1}^{\infty 1} \Delta_{m n} B_{m}=b_{n}
$$

Where

$$
\begin{aligned}
& \wedge_{n m}=d_{n-1}^{1 m}(i \alpha) \\
& \times\left(Q_{n}^{(1)}(\mathrm{i} \tau) \frac{d}{d \tau}\left(\sqrt{\left(\tau^{2}+1\right)} R_{1 m}^{(3)}(i \alpha, \tau)\right)-n(n+1) Q_{n}(\mathrm{i} \tau) R_{1 m}^{(3)}(i \alpha, \tau)\right) \\
& b_{n}=\frac{1}{\sqrt{\left(\tau_{0}^{2}+1\right)}} \delta_{n 1}
\end{aligned}
$$

\section{Pressure Distribution $P(\tau, \mathrm{t})$}

The equations that lead to the determination of pressure distribution are (3), (4), (5) and (2) and these can be rewritten as

$$
\begin{aligned}
& \frac{1}{h_{1}} \frac{\partial p}{\partial \xi}=\left(\rho i \omega+\frac{\mu}{k^{*}}\right)\left[\frac{1}{h_{2} h_{3}} \frac{\partial \psi}{\partial \eta}\right]-\frac{\mu}{h_{2} h_{3}} \frac{\partial}{\partial \eta}\left(E^{2} \psi\right) \\
& \frac{1}{h_{2}} \frac{\partial p}{\partial \eta}=\left(\rho i \omega+\frac{\mu}{k^{*}}\right)\left[\frac{-1}{h_{1} h_{3}} \frac{\partial \psi}{\partial \xi}\right]+\frac{\mu}{h_{1} h_{3}} \frac{\partial}{\partial \xi}\left(E^{2} \psi\right)
\end{aligned}
$$

Using the expression for $\psi$ in (30) and simplifying, we get $p=\frac{\mu \alpha^{2} U}{c} \sum_{n=1}^{\infty} A_{n} n(n+1) Q_{n}(i \tau) p_{n}(t)+$ An arbitrary constant

\section{Evaluation of Drag on the Spheroid}

The drag on the spheroid is given by

$\operatorname{Drag}=D_{0} e^{i \omega t}$

where

$$
D_{0}=2 \pi c^{2} \sqrt{\left(\tau_{0}^{2}+1\right)} \int_{-1}^{1}\left[t \sqrt{\left(\tau^{2}+1\right)} \mathrm{t}_{\xi \xi}-\tau \sqrt{\left(1-t^{2}\right)} \mathrm{t}_{\xi \eta}\right] d t
$$

After considerable manipulation this reduces to the seemingly simple form

$$
\begin{aligned}
& D_{0}=2 \pi c^{2} \sqrt{\tau_{0}^{2}+1} \frac{4}{3}\left[\frac{1}{2} \tau_{0} \sqrt{\tau_{0}^{2}+1}-\frac{A_{1}}{\sqrt{\tau_{0}^{2}+1}}\right] \frac{\mu U \alpha^{2}}{c} \\
&= \frac{8 \pi c^{2}}{3} \frac{\mu U \alpha^{2}}{c}\left[\frac{1}{2} \tau_{0}\left(\tau_{0}^{2}+1\right)-A_{1}\right] \\
&=\frac{8 \pi}{3} \mu U c \alpha^{2}\left[\frac{1}{2} \tau_{0}\left(\tau_{0}^{2}+1\right)-A_{1}\right] \\
& \frac{D r a g}{\frac{8 \pi}{3} \mu U c}=\alpha^{2}\left[\frac{1}{2} \tau_{0}\left(\tau_{0}^{2}+1\right)-A_{1}\right] e^{i \omega t} \\
&=\left(K_{1}-i K_{2}\right) e^{i \omega t} \\
&=\left(K_{1}-i K_{2}\right)(\cos \omega t+i \sin \omega t) \\
&=\left(K_{1} \cos \omega t+K_{2} \sin \omega t\right)+i\left(K_{1} \sin \omega t-K_{2} \cos \omega t\right)
\end{aligned}
$$

Real part of drag $=\operatorname{Re}\left[\frac{1}{2} \tau_{0}\left(\tau_{0}^{2}+1\right)-A_{1}\right] e^{i \omega t} \alpha^{2}$

\section{Numerical Work and Discussion}

To understand the variation in drag with respect to permeability parameter $\alpha$ and size of the spheroid $s_{0}$ we resort to numerical work. Since $B_{n}$ 's are zero when $n$ is even, we suppress the even suffixed $B_{n}$ 's in system (27). We truncate the system to 5 by 5 system and resort to numerical determination of $\mathrm{B}_{1}, \mathrm{~B}_{3}, \mathrm{~B}_{5}, \mathrm{~B}_{7}$, and $\mathrm{C}_{9}$ as in the earlier chapters. Then using these values and a consequence of the equation (24) with $\mathrm{n}=1$, we determine $\mathrm{A}_{1} \mathrm{nu}-$ merically. Thus, the formula in (35) allows us to estimate the drag on the spheroid numerically. For numerical evaluation we have taken

$\alpha=0.001,0.015,0.02,0.03,0.04,0.05,0.06,0.07,0.08$ 
$\tau_{0}=0.2,0.3,0.4,0.5,0.6,0.8,0.9,1.0,1.2$

and $\omega t=\frac{\pi}{12}, \frac{\pi}{6}, \frac{\pi}{4}, \frac{\pi}{3}, \frac{5 \pi}{12}, \frac{\pi}{2}$

Fig 2, 3, 4 and 5 shows the variation of drag for different values of size $\tau_{0}$ of the spheroid for fixed values of $\alpha$ and $\omega t$. We see that as the size of the spheroid increases the drag is seen to be increasing.

Fig $6,7, \& 8$ show the variation of drag for different values of $\alpha$. We note that as the permeability parameter $\alpha$ is increasing, for a fixed value of $\tau_{0}$ and $\omega t$ the drag is seen to be increasing.

Fig 9,10,11,12 and13 shows the variation of drag for different values of $\omega t$ for fixed values of $\tau_{0}$ and $\alpha$ it seems to be frequency (sine or cosine) curve.

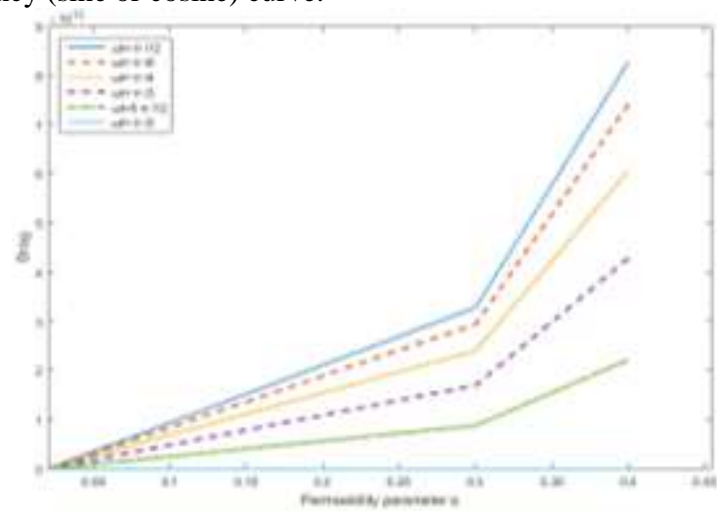

Fig. 2: Change in drag w.r.to $\alpha$ at fixed values of $\tau_{0}=0.2$ and $\omega t$.

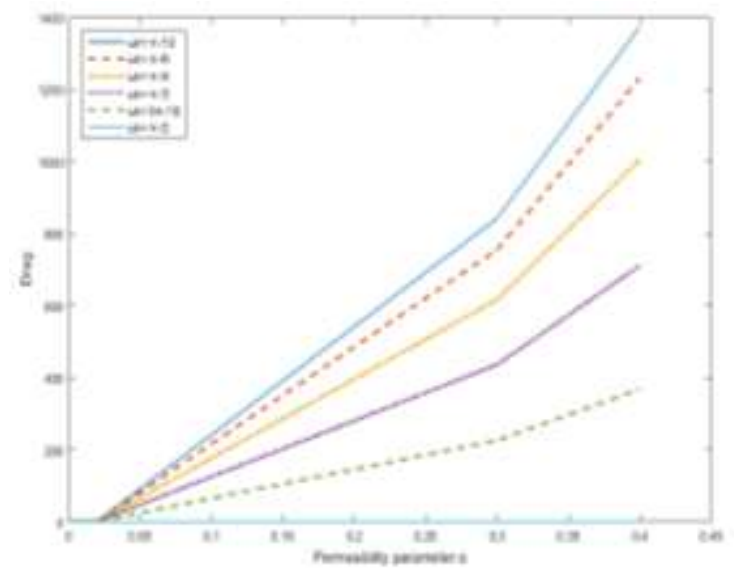

Fig. 3: Change in drag w.r.to $\alpha$ at fixed values of $\tau_{0}=0.5$ and $\omega t$

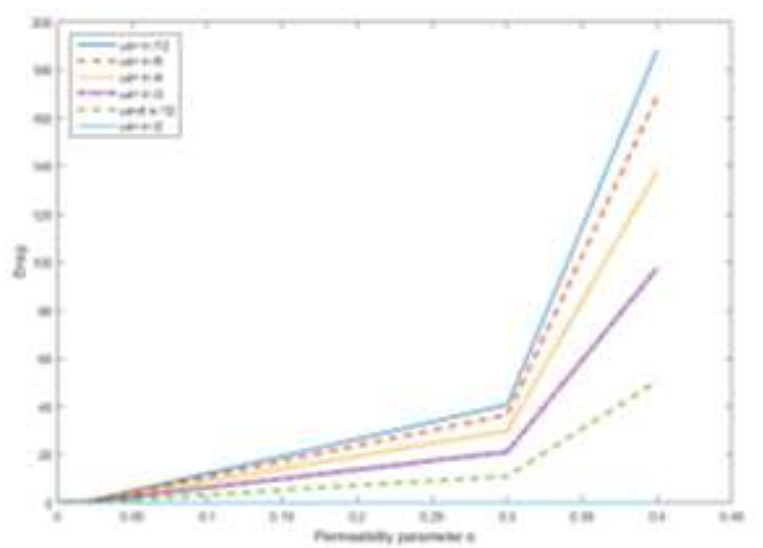

Fig 4: Change in drag w.r.to $\alpha$ at fixed values of $\tau_{0}=0.8$ and $\omega t$

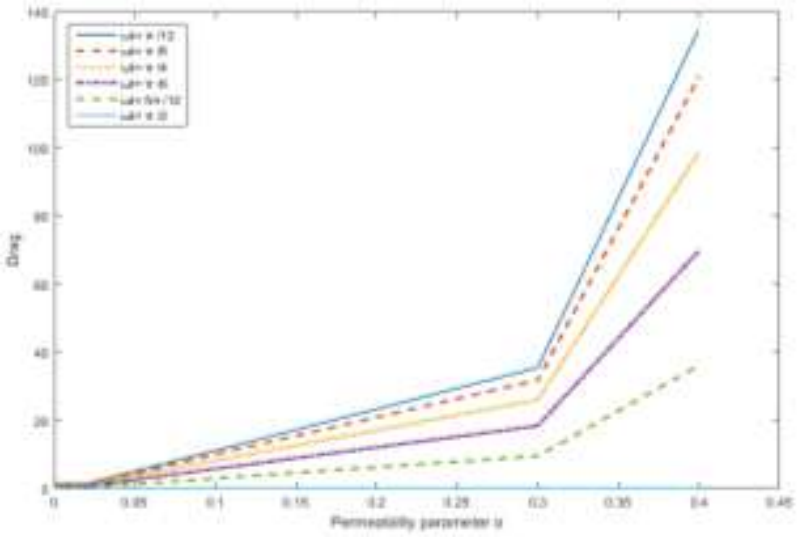

Fig. 5: Change in drag w.r.to $\alpha$ at fixed values of $\tau_{0}=1.2$ and $\omega t$

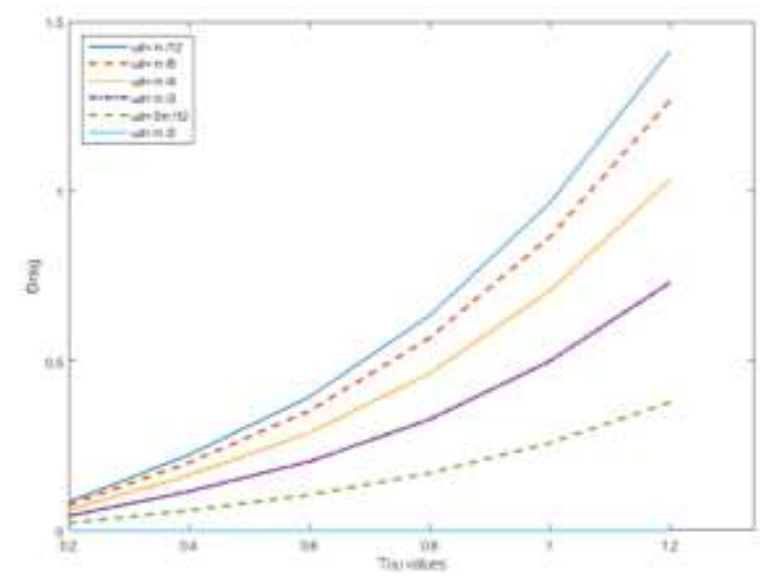

Fig. 6: Change in drag w.r.to $\tau_{0}$ at fixed values of $\alpha=0.001$ and $\omega t$.

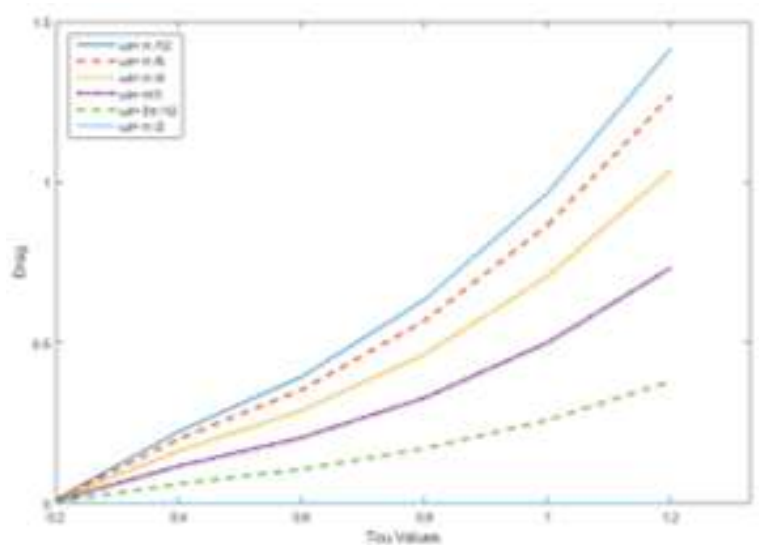

Fig. 7: Change in drag w.r.to $\tau_{0}$ at fixed values of $\alpha=0.002$ and $\omega t$

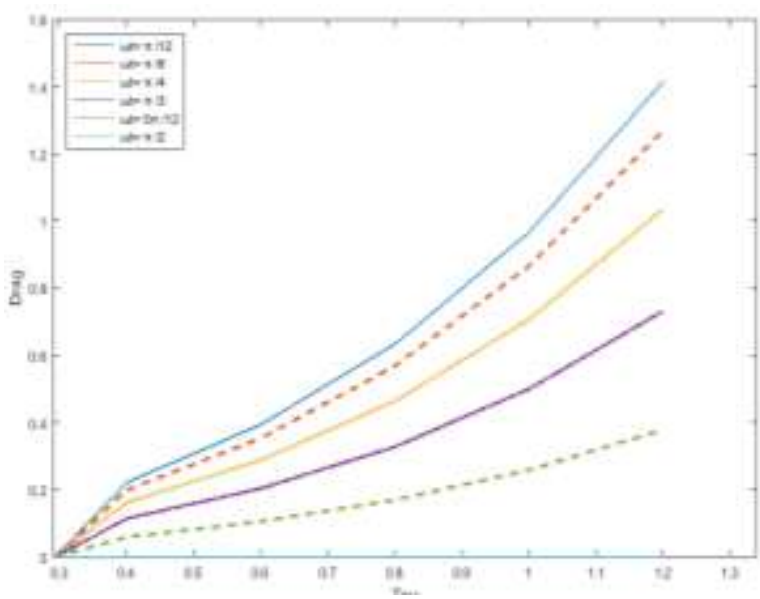

Fig 8: Change in drag w.r.to $\tau_{0}$ at fixed values of $\alpha=0.003$ and $\omega t$ 


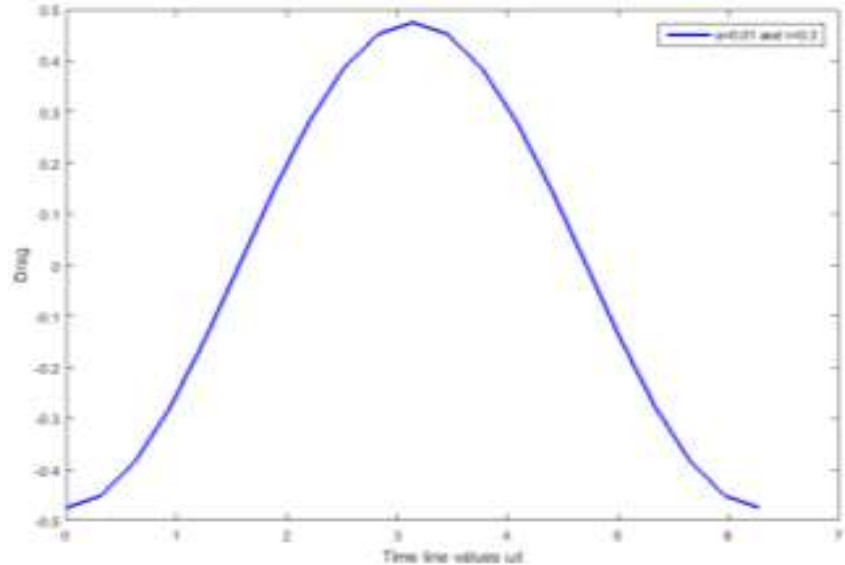

Fig. 9: Change in drag w.r.to $\omega t$ at fixed values of $\alpha=0.01$ and $\tau_{0}=0.3$

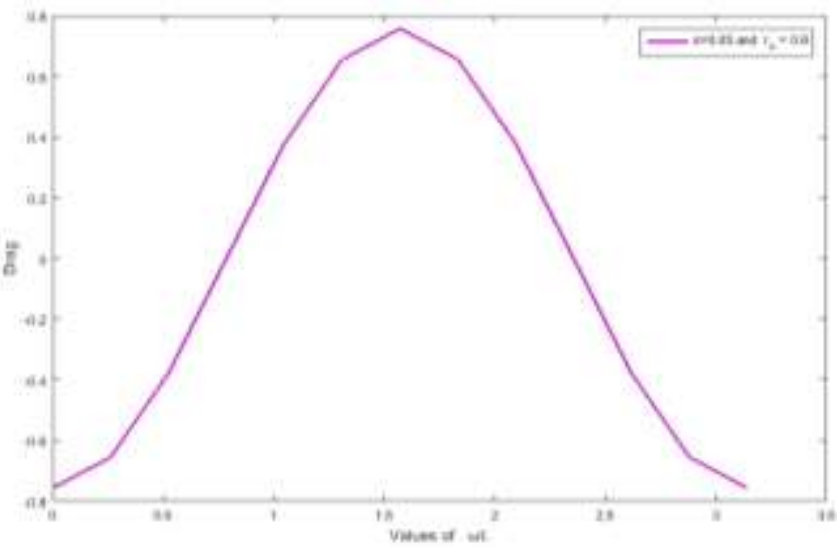

Fig. 10: Change in drag w.r.to $\omega t$ at fixed values of $\alpha=0.05$ and $\tau_{0}=0.8$

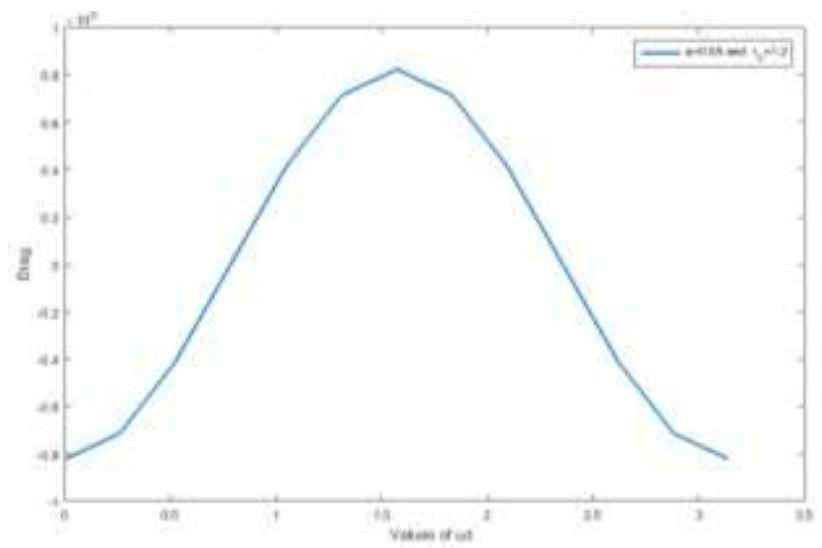

Fig. 11: Change in drag w.r.to $\omega t$ at fixed values of $\alpha=0.05$ and $\tau_{0}=1.2$

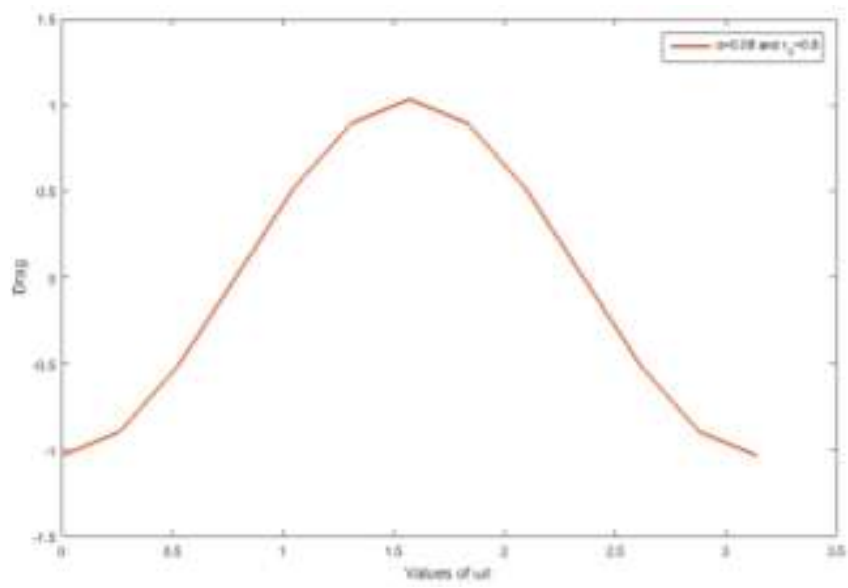

Fig. 12: Change in drag w.r.to $\omega t$ at fixed values of $\alpha=0.08$ and $\tau_{0}=0.8$.

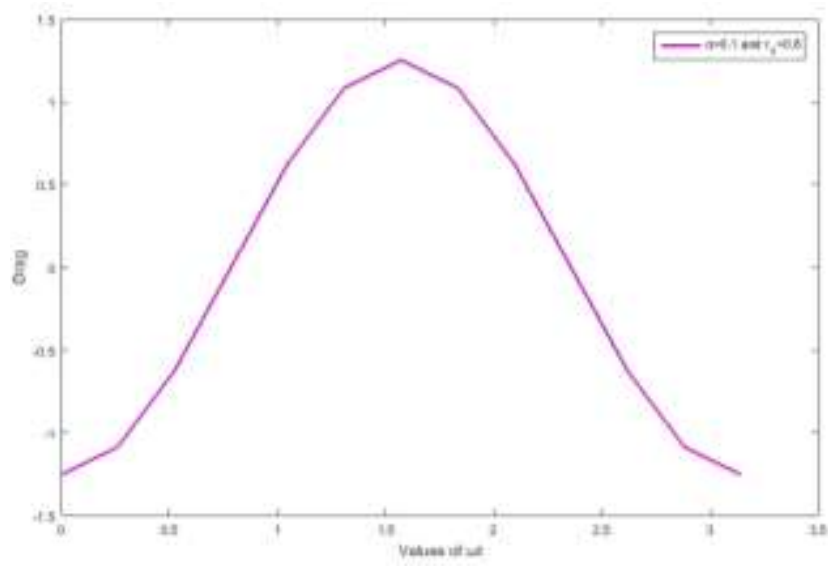

Fig. 13: Change in drag w.r.to $\omega t$ at fixed values of $\alpha=\mathbf{0 . 1}$ and $\tau_{0}=\mathbf{0 . 8}$

\section{Acknowledgement}

At outset I express my deep sense of gratitude to Prof. S. Kalesha Vali, JNTUK University college of Engineering, Viziayanagaram, who stood behind me to do this work. I would like to express my deepest appreciation to Prof. TKV Iyengar, (late) Prof. Emeritus, NIT Warangal whose sincere interest in this work and constant encouragement.

\section{References}

[1] Pozrikids.C., (2011), Introduction to Theoretical and Computational Fluid Dynamics, Second Edition, OXFORD University Press.

[2] Stokes, G. G., (1851), On the effect of fluids on the motion of pendulums, Trans. Camb. Phil. Soc, Vol. 9.

[3] Kanwal, R.P., (1955), Rotary and longitudinal oscillations of axisymmetric bodies in a viscous fluid, The Quart.J..Mech.App.Math.8, pp.146-163.

[4] Lakshmana Rao, S.K and Bhujanga Rao, P., Circular cylinder oscillating about a mean position in incompressible micropolar fluid. Int.J.Engng. Sci., Vol 10, pp.185-191,

[5] Lakshmana Rao, S.K and Bhujanga Rao, P., (1971), The oscillations of a sphere in a micropolar fluid., Int.J.Engng. Sci., Vol 9,pp.651-672.

[6] Lakshmana Rao, S.K. and Iyengar, T.K.V. , (1981), The slow stationary flow of incompressible micropolar fluid past a spheroid, Int J. Engg. Sci, Vol 19, pp 189-220.

[7] Abramowitz, M. and Stegun, I.A., (1965). Handbook of Mathematical functions with formulas, graphs and mathematical tables, Dover publications, INC, NewYork.

[8] D. Satish Kumar and Dr.S.Kalesha Vali, (2015), Brinkman Flow Past An Impervious Spheroid Under Stokesian Assumption, Global Journal of Pure and Applied Mathematics. ISSN 0973-1768 Vol 11, Number 3,pp. 1399-1412. 